\title{
LOS JÓVENES HABLAN CUANDO LAS GALLINAS MEAN": PARTICIPACIÓN Y CONTRUCCIÓN DE CIUDADANÍA JUVENIL POR MEDIO DEL ARTE EN LAS COMUNIDADES DESVENTAJADAS. LA EXPERIENCIA DE LOS/AS JÓVENES DE LA BARRIADA VENEZUELA
}

\author{
Ángel Pérez Soler ${ }^{1}$, Mónica Ponce Caballero² y Natalia Matos López ${ }^{3}$
}

\section{Resumen}

Tradicionalmente en Puerto Rico se concibe y perpetua la visión de que los/las jóvenes "hablan cuando las gallinas mean". Muchas comunidades, organizaciones sin fines de lucros y otras entidades que realizan trabajos con esta población, se limitan a realizar actividades y proyectos sin hacer parte del proceso de creación, planificación e implementación a los/las jóvenes de estos grupos. Rompiendo con estas ideas de exclusión, el Centro de Acción Urbana Comunitaria y Empresarial de la Universidad de Puerto Rico (CAUCE) y el liderato comunitario de la barriada Venezuela en Río Piedras, han trabajado en conjunto con el fin de desarrollar el liderato juvenil, iniciando un proyecto para organizar a los/ las jóvenes. El mismo ha servido de plataforma para que Las Mega Estrellas de VZ, hayan realizado diversas actividades planificadas por ellos/ellas, incluyendo un mural en mosaico, el cual adorna el centro comunal de la barrida. Este artículo intenta documentar por medio de las voces de los/las jóvenes la experiencia de estos(as) en el proyecto gestado y planificado por los/las jóvenes de la comunidad.

De igual forma, el artículo busca mostrar lo efectivo que puede ser abrir espacios de participación para jóvenes en busca de que estos(as) desarrollen estrategias y tácticas para el reclamo de sus derechos ciudadanos. A su vez, el artículo sirve para que profesionales del Trabajo Social usen las artes como metodología para la organización comunitaria y el trabajo con jóvenes. [Palabras clave: participación, planificación participativa, jóvenes y arte].

\section{Abstract}

Traditional viewpoints in Puerto Rico often conceive and perpetuate the belief that youngsters' opinions are less valuable in society. Many communities, non-profit organizations and other entities exclude their youngest members from the planning and implementation phases of events and projects. Despite these exclusionary practices that characterize many organizations, some community-based groups employ more participatory approaches. For example, the Center for Urban, Communitarian and Entrepreneurship Action (CAUCE for its Spanish acronym) from the University of Puerto Rico, along with leaders from the Venezuela Community in Río Piedras, have been working on the implementation of a project that seeks to develop young leaders and integrate them in various community initiatives. As part of this project, a group of young residents known as Las Mega Estrellas de VZ (Venezuela's Mega Stars) have been involved in different events, including the painting of a mural that now decorates the community's main meeting center. This paper seeks to document the voices and experiences of those youngsters who have been involved in the planning and creation of this project.

This paper also seeks to demonstrate the effectiveness of opening participatory spaces among the youngest members of society. Through the implementation of these strategies, this demographic sector \footnotetext{
Caribe.

${ }^{1}$ Maestro en Trabajo Social con énfasis comunitario. Doctorado del Centro de Estudios Avanzados de Puerto Rico y el

${ }^{2}$ Maestra en Trabajo Social con énfasis comunitario. Trabajadora social del Centro de Acción Urbana Comunitaria y Empresarial (CAUCE), Río Piedras.

${ }^{3}$ Maestra en Trabajo Social con énfasis comunitario.
} 
of society can become more active in the development of tactics geared at reclaiming their rights as citizens. In that sense, this research paper is a valuable tool for Social Workers involved in the use of art as a methodology for community organization and the integration of youngsters to local projects. [Keywords: participation, participative planning, youth, art].

\section{Introducción}

El presente artículo es la sistematización del trabajo realizado por los pasados años con los/as jóvenes de la barriada de Venezuela en Río Piedras, en colaboración con el componente de práctica profesional de Trabajo Social que se desarrolla en el Centro de Acción Urbana Comunitaria y Empresarial de Río Piedras (CAUCE). A su vez, el escrito busca documentar una experiencia concreta del trabajo realizado con jóvenes en Puerto Rico. Por su parte, es también la muestra más fehaciente del efecto que tienen las prácticas profesionales (en este caso las de Trabajo Social) en las comunidades en las cuales se desarrollan. Es motivo de interés documentar la experiencia, ya que CAUCE se ha distinguido por ser un espacio de taller práctico, el cual genera conocimiento basado en la realidad concreta de las comunidades aledañas al campus universitario. Son las prácticas profesionales las que nutren al estudiante de una experiencia formativa de investigación y acción en el marco del trabajo interdisciplinarios que proveen los centro de práctica (Martínez, 2009). En este caso, desde un centro adscrito a la Universidad de Puerto Rico, Recinto de Río Piedras, el cual dentro de sus responsabilidades está apoyar la gestión de los grupos comunitarios, con el fin de crear una alternativa de participación ciudadana para la rehabilitación del centro urbano y sus comunidades.

Otros de los intereses apremiantes de la investigación son el de aportar al conocimiento en la práctica con jóvenes desde la disciplina del Trabajo Social y las posibles estrategias a utilizarse para desarrollar trabajo con este sector. Esto aportará en gran medida al conocimiento desde el proceso de sistematización de las experiencias, no solamente de los y las jóvenes, sino también de las personas que acompañaron en el transcurso de las actividades.

El Centro de Acción Urbana, Comunitaria y Empresarial (CAUCE) es un proyecto adscrito a la oficina de Rectoría de la Universidad de Puerto Rico, Recinto de Río Piedras. En el mismo se promueve la rehabilitación social, física y económica del casco urbano de Río Piedras y de las comunidades aledañas (Buen Consejo, Capetillo, Santa Rita, Blondet, García Ubarri, Venezuela y el casco urbano, incluyendo el Paseo de Diego, Plaza de Convalecencia, Plaza Robles, Plaza del Mercado y las calles cercanas). Algunas de las tareas realizadas por CAUCE son: fortalecer el desarrollo organizativo de los residentes de las distintas comunidades; atender situaciones particulares que atañen la vida social y económica de Río Piedras; servir de puente entre las agencias de gobierno estatales y municipales y los grupos de residentes organizados; servir de apoyo en las iniciativas comunitarias (deportivas, culturales, económicas y organizacionales).

Dentro de las iniciativas comunitarias que se han atendido, en colaboración con el liderato comunitario, se encuentra el desarrollo de grupos de niños/as y jóvenes por distintos sectores. El foco de estudio es el grupo de niños/as y jóvenes iniciado en 2012 en la barriada de Venezuela, el cual lleva el nombre de Las Mega Estrellas de Venezuela. Este grupo se compone de alrededor de quince a veinte jóvenes activos/as, de siete a dieciocho años de edad, que se reúnen semanalmente utilizando como sede el centro comunal de la barriada. Dentro las tareas realizadas por el grupo se destacan las bicicletadas, cine foros y talleres de diversos de temas. $Y$ su objetivo es demostrar que en la comunidad hay un grupo activo y ansioso por trabajar en iniciativas que promuevan la autogestión en la zona y desarrollar su liderato comunitario.

Su proyecto cumbre fue la elaboración de un mural en mosaico en la pared del centro comunal de la barriada Venezuela. Sin duda, al momento, es el más grande realizado por el grupo. La confección comenzó el 29 de marzo del 2014 y se trabajó todos los fines de semana, durante un mes y medio, lo que se traduce en varias horas de trabajo autogestionado. El producto de esta gestión buscaba la apropiación del espacio y la reafirmación de la identidad juvenil dentro de la comunidad. Esto desde la experiencia de vida de un sector (los/as jóvenes) que ha sido marginado por nuestra sociedad. Además, la construcción del mural refleja una postura crítica de parte de un grupo de jóvenes que busca el reconocimiento como ciudadanos y ciudadanas y la activación social de una propuesta alterna que sirvió para reafirmar y consolidar el impacto de este grupo en la comunidad, tomando en cuenta la 
heterogeneidad del mismo. Por lo tanto, la utilización del arte fungió como vehículo para adelantar y reafirmar los procesos organizativos entre los y las jóvenes y visibilizaron el mensaje que se propusieron llevar.

El arte se ha utilizado para fomentar la unidad en las comunidades y grupos. Esta herramienta se emplea para desarrollar y fomentar el trabajo en colectivo, mientras se crea la obra artística, además ha servido como vehículo de expresión artística el cual funge como un vocero de comunidades y grupos marginados o minoritarios. El mismo tiene el efecto de retomar los espacios perdidos y utilizarlos con el fin de llevar un mensaje. También funciona como la perpetuación de la memoria, ya que plasma en colores y diferentes materiales, como fotografía y losetas, la trayectoria de una comunidad o un grupo en un espacio sociocultural específico. El hacer memoria pasa a integrar, de manera primordial, un concepto mayor llamado identidad, la que puede ser grupal o individual; la que indica, a su vez, un sentido de permanencia tanto en el campo temporal como en el espacial (Alcatruz, 2004, p.4). En este proceso de asimilación e internalización del sentido de pertenencia por medio del mural, los y las jóvenes desarrollan simultáneamente destrezas tales como el trabajo en equipo, liderazgo, cooperación, participación, planificación y la elevación del carácter ciudadano de los/as individuos. Por otra parte, este esfuerzo ayuda a desarrollar la creatividad e imaginación de sus participantes creando un ambiente dinámico entre el grupo.

\section{Objetivos del estudio}

Este artículo tiene como objetivo el describir y analizar el proyecto del mural-mosaico a través de la experiencia de los/as jóvenes que componen el grupo en la barriada Venezuela, siendo ellos/as el eje central de la iniciativa. Con esto intentamos visibilizar el trabajo que hicieron al rescatar el espacio dentro de su comunidad, convirtiéndolo en un espacio colectivo. Por otro lado, aportamos a la discusión sobre la importancia de la participación juvenil en los procesos comunitarios rompiendo con los mitos populares como: "Los niños hablan cuando las gallinas mean" o "La juventud está perdida". Discutiremos la gama de posibilidades que brinda el arte como medio de organización comunitaria y de acción social con los/as jóvenes.

\section{Revisión de literatura}

El arte como herramienta de impacto social. El arte en todas sus manifestaciones es considerado unas de las herramientas de expresión más completas y complejas. Por mucho tiempo las artes eran consideradas disciplinas culturales de poblaciones que estaban más expuestas a una educación formal. En aquel entonces, las artes se limitaban a personas que tenían las posibilidades de visitar museos, teatros, galerías y otros espacios de muy exclusivo acceso. Con el tiempo, algunos/as artistas han trabajado arduamente para romper con esas concepciones. Una de las disciplinas del arte que ha roto con ese espacio de elite ha sido el "arte callejero". El arte callejero se manifiesta en diversos contextos, el cual tiene como objetivo ser expuesto en lugares públicos, por lo general muy transitados, con el fin de cautivar al público espectador y visibilizar el mensaje que quiere plasmar su creador o creadora.

Otra de las prácticas artísticas de resistencia a la tradicional forma de arte elitista es el "arte comunitario", el cual se considera como un:

(...) conjunto de iniciativas a partir de la colaboración entre artistas y las comunidades locales, cuyo objetivo es expresar, a través del arte, identidades, preocupaciones e ideas, a la vez construir capacidades culturales y contribuir al cambio social (Suess, 2006, p.70).

Esta movilización artística dentro del espacio, promueve gestar proyectos colectivos que traen consigo experiencias organizativas y creativas en las comunidades. Según Nardone (2010), el concepto de "arte comunitario" surge principalmente de países anglosajones. "Se utiliza para describir prácticas artísticas de naturaleza grupal, que implican la participación en un proceso creativo y su desarrollo en la comunidad" (Nardone, 2010, p.47). Aunque existen distintas versiones de su aparición, en varios escritos concuerdan, que este tipo de arte surge gracias a artistas con inquietudes sociales. "Estos artistas se acercaban a las comunidades marginadas para llevar sus proyectos artísticos; de esta forma se distanciaban de un ambiente artístico establecido" (Suess, 2006, p.71). Por otro lado, dentro de los 
objetivos subyacentes se encontraba el "mejorar los espacios públicos donde vivía la clase trabajadora, promoviendo que fueran los propios residentes, agentes activos en la transformación de ese entorno" (Palacios Garrido, 2009, p.203).

Para cumplir con el objetivo de abrir nuevos espacios artísticos se organizó un grupo de artistas comprometidos/as con el cambio social, enfocados/as a exponer a las personas de los barrios a una nueva noción de arte, rompiendo con la visión de las artes como herramientas única de las elites que se exponen sólo en los museos importantes.

El cuestionamiento tanto del arte por el arte como de los valores del formalismo, o del sistema del mercado artístico, propició una deriva hacia la idea de la obra de arte como herramienta para el cambio social. La crítica del individualismo como eje del proceso creativo y del artista como creador aislado y genial favoreció que un número importante de artistas renunciasen a su estatus y cambiasen un marco de elitismo cultural por una vinculación de su trabajo a problemáticas del contexto social en el que se desenvolvían sus vidas. Normalmente en relación a grupos sociales desfavorecidos y a sus necesidades. Esta importancia por el contexto llevó a sacar a la obra de arte de la galería y del museo y a situarla en ámbitos más cotidianos (Palacios Garrido, 2009, p.199).

El primer acercamiento al "arte comunitario" se enfocaba sólo en "dar" arte a las comunidades. Para la época de los años ochenta el paradigma tomó un nuevo rumbo y se encaminó a reconocer la multiplicidad de posibilidades que existían en estos espacios comunitarios y la posibilidad de creación de proyectos autónomos. Esta nueva visión sostenía que las comunidades desventajadas tenían una cultura particular, aunque fuera marginada por la cultura dominante. Esto ayudó a redefinir el rol del artista, pasando a ser denominado como "trabajador del arte" (Pitts \& Watt, 2001).

Luego de pasar por los cambios descritos, en la década de los noventa las intervenciones de los/as artistas en las comunidades acuñaron un nuevo término proveniente de Europa, el cual se conoce como animadores socioculturales. "La función central del desarrollo cultural comunitario pasó de ser la producción de arte, a ser más bien consolidación y desarrollo de la comunidades. El cambio fue uno significativo, de ser las comunidades objeto de los proyectos, ahora serían parte del mismo" (Pitts y Watt, 2001, p.10).

Estos cambios metodológicos del arte callejero han fortalecido el concepto, dando espacio a una mayor autonomía en los procesos creativos de las comunidades, respetando sus multiplicidades y provocando la realización de proyectos artísticos de diversas áreas como lo son: teatro, música, danza y murales. El artista, funge como facilitador/a, entendiendo y aceptando la creatividad y la autonomía de la comunidad en la elaboración de una obra artística. Por lo tanto, el arte y sus creadores/as no son pasivos/as, individuales y neutrales, por el contrario, construyen formas creativas para la acción social, promoviendo el trabajo colectivo y asumiendo unas posturas contundentes ante problemáticas sociales particulares.

Por otra parte, además de ser un medio para provocar la acción y movilización, el arte callejero ha sido una herramienta importante para denunciar los problemas provocados por la cuestión social. Desde rescatar espacios abandonados y organizar grupos en las comunidades, hasta provocar discusiones críticas. El ejemplo más notable fue la utilización del muralismo en México luego de la Revolución Mexicana. Es bajo el mandato del Estado, y con iniciativas de David Alfaro Siqueiros, Diego Rivera, José Clemente y Rufino Tamayo, que el muralismo comenzó a servir de comunicador de asuntos sociales. A través de los murales, la Escuela Muralista Mexicana se convirtió en un movimiento político y social de resistencia e identidad donde se exponían temas como la revolución, lucha de clases e luchas indígenas (Alfonso Pérez, s.f.).

Otra manifestación de las artes contestatarias fue la de Augusto Boal con su teatro del oprimido. Este buscaba, a través del arte escénico, mejorar la calidad de vida de grupos sociales e individuos estigmatizados. El concepto consistía en utilizar a personas que no eran actores, ni actrices, facilitando la expresión y la creatividad de los mismos y las mismas. A eso le podríamos sumar un sinnúmero de iniciativas enfocadas a un cambio social radical.

(...) las propuestas basadas en el "arte transformador" pueden promover la participación en cuestiones que hacen al desarrollo de la comunidad, y pueden ser vistas como catalizadoras de cambios a nivel personal, grupal y comunitario, generando así otras alternativas y campos de participación (Roitter, 2009, p.2). 
Si bien estas iniciativas creativas provocan dinámicas sociales en el momento de realizarse, es importante que no se limiten a ser proyectos momentáneos, sino que provoquen experiencias movilizadoras, educativas y liberadoras. Para que estas acciones se lleven a cabo, hay que aprovechar la coyuntura organizativa en el proceso de creación artística, donde los grupos pueden continuar trabajando en las problemáticas dentro de la comunidad, fortaleciendo su liderato, la autogestión y la participación ciudadana.

Al momento de realizar proyectos de impacto en las comunidades, el llamado arte comunitario busca provocar el rescate de espacios baldíos, reclutar para la organización, sensibilizar a los y las participantes y organizar otras movilizaciones. El arte comunitario es una forma de promover la participación grupal de una manera no tradicional, con la cual se puede integrar personas de todas las edades y ofrecer experiencias innovadoras de expresión comunitaria. A su vez, es una forma de elevar el carácter ciudadano de personas que no tienen espacios de participación en la sociedad (Pizarro, 2012). Es por ello que el profesional del Trabajo Social debe considerar el arte en todas sus manifestaciones como una herramienta en su proceso de apoyo dentro de los espacios de trabajo, aliándose de forma interdisciplinaria con artistas que puedan concretizar proyectos donde las personas sean el eje central. Con estas manifestaciones artísticas se construye una identidad colectiva autogestionada por parte de los/as vinculados/as a la comunidad, dando a su vez espacio a la expresión individual.

Sentido de pertenencia (retomar el espacio público-comunitario). El espacio comunitario es uno de los lugares más inmediatos, donde las personas se interrelacionan a partir de elemento compartidos, incluyendo el territorio geográfico. Según los estudiosos/as de las materias sociales una comunidad es:

(...) una agrupación o conjunto de personas que habitan un espacio geográfico delimitado y delimitable, cuyos miembros tienen conciencia de pertenencia o de identificación con algún símbolo local y que interaccionan entre sí más intensamente que en otro contexto, operando en redes de comunicación, intereses y apoyo mutuo, con el propósito de alcanzar determinados objetivos, satisfacer necesidades, resolver problemas o desempeñar funciones sociales relevantes (Sánchez \& González, 2006, pp.33-34).

Añade Tonon (2009, p.18) que esa comunidad tiene elementos ciudadanos y que la ciudadanía no se puede limitar a un concepto jurídico, sino que se arraiga a expresiones de identidad y pertenencia, lo que lo hace eminentemente político. Es aquí donde se estructuran y desarrollan actividades como la participación y la acción social, que funcionan a partir de relaciones complejas dentro y fuera de la comunidad. Estos espacios, delimitados geográficamente, funcionan como un elemento de unión entre los individuos y familias que los integran. Según De Pablos y Sánchez (2003, p.13), se parte de la consideración de que el espacio habitado tiene una significación para cada persona y cada grupo social, además de que todo barrio posee condiciones particulares que le hacen distinguirse de otros. En estos espacios las personas van creando vínculos entre sí, interaccionando y compartiendo experiencias de vida, valores y redes de apoyo inmediatos. Los lugares dejan de ser territorios de paso y se transforman en áreas de encuentros donde se desarrolla la historia y la memoria de los sujetos.

Las comunidades son dinámicas, contenidos en ella la heterogeneidad y la diversidad, donde va evolucionando a partir de elementos políticos, sociales, económicos que inciden dentro y fuera de estos. Estas áreas geográficas son el reflejo del paso de la historia, conglomerando así múltiples realidades que nos muestran la situación actual, que en muchas veces, terminan como espacios en el olvido.

En la búsqueda de la rehabilitación de un espacio es necesaria la utilización de varios elementos para darle continuidad al trabajo de la comunidad. Sánchez y González (2006), nos hablan de:

(...) la necesidad de revitalizar un espacio social urbano, tomando como eje fundamental quienes lo habitan, obliga detenerse en la detección de los factores que constituyen los elementos claves de la percepción del espacio habitado, como variante significativa en la búsqueda de calidad de vida. (p. 20)

Por tanto, se desarrollan actividades que sean cónsonas con los objetivos de la comunidad, ya sea para resaltar su belleza, reclamar el espacio, dar cohesión al grupo o que sirva como acto de 
afirmación de identidad. Esta identidad de los/as sujetos dentro del espacio compartido, es afectada por las políticas sociales y económicas acuñadas por el sistema en que vivimos. Por lo tanto, surge la necesidad de crear procesos que conlleven a la participación colectiva dentro de las comunidades, con el fin de impulsar una movilización social, donde la afirmación del espacio juega un papel importante. Los/as jóvenes son claves para la continuidad y desarrollo de este proceso, deconstruyendo la noción de los/as jóvenes como un grupo ausente en los procesos de cambio es sus comunidades.

Otro asunto importante en el rescate de los espacios públicos es exponer el carácter real de lo "público". La mejor forma de describir lo antes expuesto nos la señala el literato puertorriqueño Eduardo Lalo (2005), cuando nos dice:

Una vez más, la ciudad se ha fraguado hacia dentro: espacios refrescados en el mundo privado de los ventiladores y acondicionadores de aire. Una vez más se privilegia los espacios egocéntricos sobre el colectivo. San Juan no ha sabido crear sus espacios públicos, sus alamedas, sus paseos, bosques, parques. Los pocos que existen muchas veces se encuentran desiertos, porque San Juan no sabe vivir puertas afuera y se extienden cada día más su medievalización de sistema de seguridad, rejas, murallas, controles de acceso, policía privada. (p.26)

Lalo (2005), nos ofrece una descripción de cómo se ha fraguado el espacio público, en la ciudad de San Juan, asunto que se refleja de igual forma en Río Piedras. Como se explicó anteriormente: los espacios públicos no tienen carácter de público, si no hay una apropiación por parte de las personas. Es por esto que carecemos de espacios donde se pueda compartir ideas que propendan al verdadero ejercicio democrático y ciudadano. El privilegiar los espacios privados es, en gran medida, una manifestación del sistema en el que la sociedad puertorriqueña se ha construido. Vale la pena realizar una observación al libro del arquitecto Edwin Quiles (2009), La ciudad de los balcones, y comparar su contenido con las fachadas realizadas por las nuevas construcciones para observar el cambio que se ha fomentado. La nueva construcción ha abandonado las fachadas de balcón, espacios que históricamente han servido para la confraternización, diálogo y encuentro, prácticas que fomentaban la discusión política amplia, no partidista, y que, a su vez, ampliaban la discusión ciudadana de aquellos asuntos. De igual manera, esto ha sucedido con las plazas públicas, parques y otros espacios relacionados, los cuales se conciben desde lo público, en contraposición al espacio íntimo que privilegia la propiedad privada (Fontánez, 2014, p.78). Estos espacios son conocidos en derecho como espacios públicos tradicionales. Y se consideran como lugares donde los/as ciudadanos/as se encuentran para el debate público, la reunión y los encuentros. El problema de los espacios públicos tradicionales ha sido que el carácter público de los mismos se ha visto reducido por la falta de apropiación ciudadana. En gran medida, esto responde a la seguridad relativa que ofrecen los espacios privados y limitados. Muchas veces, los espacios privados y limitados responden a una lógica mercantilista, la cual está enmarcada en el lucro de sectores específicos. Es de esta forma que la ciudadanía relaciona lo privado con la seguridad y lo público con el peligro. Este particular se puede observar cuando se comparan los centros comerciales con las plazas públicas. Al centro comercial tener una intención mercantil a sus espaldas, fomenta un ambiente de seguridad, para preservar y canalizar la compra y venta de la mercancía ofrecida. De lo contrario, la plaza pública, las calles y los parques son espacios donde no existe dicho interés y la seguridad se limita a la frecuencia y cantidad de usuarios del espacio. Fontánez (2014), señala que, a pesar de que los centros comerciales son espacios donde permea la inversión privada, los mismos han pasado a tener un carácter semipúblico, ya que el interés mercantil los ha convertido en lugares de encuentro por excelencia, suplantando así a los espacios públicos tradicionales. No obstante, el propio Tribunal Supremo de Puerto Rico, ha limitado las acciones que se pueden realizar en los espacios públicos. Y es que la demarcación de lo público desde el derecho, utilizando categorías legales, tiende a limitar la verdadera libertad de expresión. El discurso legal del espacio público simplifica las miradas y limita la racionalidad de los acontecimientos de los cuales los/as ciudadanos/as manejan en su diario vivir. Dicho de otro modo, el derecho busca dar definiciones neutras, usando principios universales que, en última instancia, limitan la libertad de expresión que se desarrolla en los espacios públicos. En síntesis, no existen espacios públicos verdaderos definidos en derecho y que si los/as ciudadanos/as no se apropian de los mismos, particularmente los conocidos como tradicionales, no habrán lugares para la discusión libre y democrática, lo que limitará el ejercicio democrático de nuestra sociedad. 
La participación de los/as jóvenes en los procesos sociales. Según María Paula Romo (2005), la inserción de la juventud en la vida política y democrática del siglo XXI, se manifiesta de formas no tradicionales:

Los/as jóvenes, hombres y mujeres, hoy muestran, mostramos, nuevas formas de hacer política, de activismo, de resistencia: el arte (desde la música, la literatura o el teatro hasta el grafiti) es un mecanismo de denuncia y protesta; allí podemos encontrar y comprender muchas de las opiniones políticas de los jóvenes latinoamericanos de hoy. (p.110)

Es que, durante mucho tiempo se ha escuchado sobre la apatía de la juventud a la organización y la participación en los procesos sociales y políticos. Sin duda, el escenario comparativo de lo que fue la militancia de los/as jóvenes de las décadas del 1960 y 1970 es muy diferente a la militancia de los/as jóvenes en la actualidad. Para el/la joven de las décadas del 1960 y 1970, el término militancia estaba vinculado a la jerarquía, disciplina, verticalidad y al constructo de un artefacto cerrado, difícil del permear (Balardini, 2005). Siendo lo antes expuesto un espacio poco simpático, el/la joven del siglo XXI, busca expresar su sentir en estructuras informales, donde el compromiso no esté encerrado en una estructura moral y que, a la misma vez, le permita sentir libertad y sea acompañado de un sentimiento de placer. Si nos amparamos en la descripción de las nuevas formas de organización de la juventud, es fácil derrotar la constante de que al joven no le interesan las cuestiones sociales y que se encuentran ajenos a los problemas que enfrenta la sociedad. Por el contrario, si algún sector se ve más azotado por las políticas neoliberales que imperan en nuestro sistema, es el sector juvenil (Rodríguez, 2005). Hay que recordar que la juventud es un periodo transitorio en la vida en el cual se perfila el futuro ciudadano/a. Valdría la pena reflexionar cuántos espacios verdaderamente democráticos existen para el desarrollo de proyectos juveniles de largo alcance. Es así que el/la joven se ve limitado a expresar su forma de pensar y su análisis de lo que debe ser las posibles soluciones a la cuestión social. La atención a este sector de la población se limita a señalarlos sólo cuando alteran las estructuras del orden establecido. "Los muchachos del punto", "la muerte de un joven" y "esos chamaquitos no saben" son sólo algunas de las expresiones que escuchamos, cuando algún joven decide retar las normas de lo establecido. A partir de esa visión negativa de la juventud, nacen políticas sectoriales, que lo único que buscan es resolver unos asuntos puntuales, reactivos y reductos que crean mayor apatía en la población. Es así que vemos constantes políticas que evocan a la urgencia y que resultan contradictorias y coyunturales. Por otro lado, cuando hay algún tipo de acercamiento del Estado, que se enmarca en el derecho ciudadano de los/las jóvenes, podemos observar el interés de los partidos políticos en buscar el adepto juvenil para nutrir su base electoral y proseguir con las políticas ante mencionadas. Esto sucede constantemente en las escuelas superiores del País, cuando se acerca el periodo eleccionario, donde un contingente de funcionarios/as públicos llega a los planteles escolares buscando que los/as jóvenes tramiten su tarjeta electoral. Si bien es cierto que la participación electoral es un derecho fundamental en la estructuración de la democracia actual, la misma no es sinónimo de un verdadero proceso participativo consciente. La participación juvenil en las elecciones termina siendo un ejercicio poco informado, el cual limita la toma de una decisión ciudadana. En Puerto Rico lo vemos a cada cuatro años, cuando parte de las consideraciones de mayor peso es la estética de candidatos y su proyección mediática. Esto dejando a un lado cualquier consideración de tipo político e ideológico. Es por esto que urge una política activa de ciudadanía juvenil, la cual permita la participación real en procesos sociales para dicho sector. La única forma posible de construir dicha ciudadanía juvenil es haciendo parte a los/as jóvenes en la construcción de las políticas que se vayan a implementar. Sin la verdadera participación de los/as jóvenes en la construcción de los proyectos, será imposible superar los resultados obtenidos hasta el momento. Es importante que, a la hora de trabajar con la población joven, no se limite a escribir una ley, tratado, entendido o reglamento. Estos esfuerzos deben estar acompañados de planes integrales que apuesten a la formación ciudadana y no a la transmisión de saberes. De lo que hablamos es de educar a los/as jóvenes sobre el mundo del cual ellos/as van a ser parte en escaso tiempo y proveer posibles soluciones, desde su perspectiva de ciudadanos/as, que ayuden a su inserción dentro ese nuevo mundo soñado. Rodríguez (2005), nos dice:

(...) consolidar políticas y programas que permitan mejorar la inserción social de las y los jóvenes (en tanto tales), sino que se orienten a tratar de mejorar el mundo al que les va a tocar integrarse 
(cuando sean adultos), algo totalmente lógico si se asume la transitoriedad como una regla básica. (p. 64)

Esa tarea le corresponde, no al joven -que como bien explica Rodríguez (2005), está en una etapa de transición-, sino al adulto que ya es parte de la vida social y que es capaz de identificar cuáles cambios sociales son a largo plazo. Si bien es cierto que los/as jóvenes del siglo XXI, son menos simpáticos a la organización tradicional, enmarcada en la lógica del partido (presidente, vicepresidente, tesorero, secretario, etc.), se deben buscar nuevas formas de organización. Es por eso que uno de los escenarios que más adeptos tiene entre los/as jóvenes son las luchas reivindicativas inmediatas, como la ampliación de los derechos a la comunidad LGBTT, las luchas ambientales y otras, asuntos que se despegan un tanto de la lucha histórica de la contradicción entre el capital y trabajo. A pesar de que las antes mencionadas tienen un alto contenido de la contradicción capital/trabajo, las mismas se visten de unos problemas inmediatos que pueden tener solución a corto plazo, como lo es la defensa de un espacio público (playa, parque u otro), la aceptación a la diversidad o la denuncia de la cuestión social por medio del arte. La razón de lo antes expuesto es que muchos/as jóvenes buscan hacer instancias concretas, donde su esfuerzo tenga resultados tangibles y puntuales. No les preocupa el saldo organizativo, sino el saldo resolutivo. Es responsabilidad del adulto hacer de esas experiencias resolutivas, instancias organizativas de futuro. La visión de los/as jóvenes es una muy "natural", es la relación entre el trabajo y la producción. A todo trabajador/a le interesa ver su trabajo completado. El mismo crea una satisfacción personal vinculada a la producción, cosa que el sistema capitalista ha desarticulado al estar la ganancia en manos de los/as propietarios/as y no en manos de los/as trabajadores/as. La misma lógica se emplea en la construcción de un proyecto social, en el cual, como la ganancia es intangible, la satisfacción de la participación es lo que se convierte en ganancia.

De esta forma, se hace imperante la implantación de proyectos que respondan a las inquietudes de los/las jóvenes, desde una perspectiva juvenil, y que los/as mismos/as estén basados en el respeto y aceptación de la visión de estos/as. Para realizar una aportación en esta dirección, valdría la pena hacer un análisis de las estructuras en las cuales participan los/as jóvenes en nuestra sociedad (escuelas, colegio, clubes y espacios deportivos). Es importante desarrollar una ciudadanía juvenil, ya que vivimos un momento de transición mundial donde la generación que vive la juventud en pleno siglo XXI, será la que moverá los asuntos sociales, políticos y económicos en muy poco, dentro de una nueva lógica tecnológica, la cual es más conocida por esta generación que por la generación que dejó atrás su juventud en las décadas anteriores al 1990.

\section{Metodología}

Para propósitos de la investigación se escogieron siete jóvenes. Los criterios para la participación en las entrevistas fueron los siguientes: vivir en la barriada Venezuela de Río Piedras, pertenecer al grupo de jóvenes "Las Mega Estrellas de Venezuela" y haber contribuido en la creación del mural. El proceso de reclutamiento de los/las jóvenes se realizó por disponibilidad. Por ser menores de edad, previo a la entrevista, se habló con cada madre, padre o encargado/a para pedir aprobación para participar en el estudio. Las personas encargadas firmaron una hoja de consentimiento. A los/las jóvenes que aceptaron participar, se les explicó en qué consistía el estudio y las preguntas que contenía la entrevista, y se les entregó la hoja de consentimiento que también firmaron.

Para la recopilación de datos, desde el diseño fenomenológico, utilizamos entrevistas semiestructuradas con el grupo de participantes. Esta entrevista se llevó a cabo en el Centro Comunal de la Barriada Venezuela. A cada una de los/as jóvenes se les entrevistó individualmente con una guía de siete preguntas semiestructurada. Escogimos este tipo de estrategia para recoger los datos ya que nos ofrece flexibilidad y dinamismo. Además, asegura la confidencialidad, protección y confianza de los y las participantes, elementos que pudieran verse fragilizados por la temática que nos ocupa en esta investigación. Según Álvarez-Gayou (2007) citado en Burgos (2011) indica que este tipo de entrevista contiene una serie de temas y algunas preguntas sugeridas, pero presenta apertura para cambios en la secuencia de la entrevista y forma de realizar las mismas de acuerdo a la situación de las personas entrevistadas. Era de nuestro interés poder integrar preguntas que surgieran en la interacción de la entrevista, mostrando así las flexibilidades de la misma. 
Para mantener la privacidad de estos datos, sólo tendrán acceso el investigador y las investigadoras. Luego de las entrevistas, las mismas se guardarán hasta tres años después de haber empezado la investigación. Luego de esta fecha, se borrará toda información grabada y se desecharán. De igual forma, utilizamos la primera letra del nombre y primer apellido del participante para salvaguardar la confidencialidad.

Como parte del plan de análisis describimos las categorías y proseguimos a transcribir todas las entrevistas, para luego hacer un doble cotejo de las narrativas. Posterior a eso, nos dimos a la tarea de realizar los análisis correspondientes utilizando el método tradicional. Los datos recopilados, los estudiamos a través del análisis de contenido y el uso de una categorización previa relacionada a los objetivos de la investigación. Sin embargo, realizamos una identificación de temáticas emergentes como parte del análisis. Para el análisis de las narrativas de los y las participantes se utilizó el modelo de análisis de Harry F. Wolcott. Este modelo, según Lucca y Berríos (2003), consta de tres partes: a) la descripción, b) el análisis, y c) la interpretación. La descripción de refiere al relato definido por las investigadoras como ocurrió y se observó. En esta etapa observamos actitud y el comportamiento de los/as participantes desde el inicio. El segundo elemento de este modelo es el análisis. Según Wolcott (1994, p. 24) "el análisis se refiere específicamente a los procedimientos sistemáticos con el fin de identificar las características esenciales y las relaciones de consonantes con los descriptores." Luego comenzamos a codificar en el cual se tomaron fragmentos de las narrativas de los y las participantes que entendíamos que se adjudicaban a las categorías previamente construidas.

Como ya fue señalado, las preguntas que se realizaron a los/as jóvenes de la comunidad Venezuela buscaban conocer a fondo temas como participación juvenil, experiencias, frustraciones, trabajo en equipo y procesos organizativos que se desprendieron del proyecto. Esto se analizó buscando la importancia, beneficios, formas de organización y sentimientos de los/as jóvenes en el proceso. Las narrativas se codificaron en varias categorías que son: el sentimiento de los/as jóvenes durante el proceso, proceso de organización del grupo, planificación participativa y liderato comunitario.

Sentimientos de los/as jóvenes en el proceso y reflexión sobre el trabajo realizado. En el proceso de crecimiento y desarrollo personal, las personas pasan por situaciones que marcan la preferencia y en ocasiones la ruta que marcarán los individuos. Es por eso que iniciamos las entrevistas con dos preguntas básicas; ¿Cómo te sentiste en el proceso del mural? ¿Qué piensas del mural? Algunas de las contestaciones marcan la razón de ser de este proyecto. N.O, expresó:

Cada vez que lo veo es un orgullo. Es una huella que nosotros dejamos en el barrio. Porque se llevó la idea del mural fue pa' que el barrio viera los jóvenes trabajando. Y al lograrlo y que se viera la huella es un orgullo, para mi cada vez que lo pienso.

Por su parte, T.B relata: Yo pienso que es algo inspirante. Porque las personas lo ven y durante el tiempo que hemos pasado después del mural ha estado llegando gente nueva a este grupo.

Otro de los que dijo su sentir fue J.D, quien señaló:

Pues mano estuvo divertido, porque tuvimos que pintar, con pintura de spray. El mosaico me cortó un poquito, pero tuvimos que trabajar en equipo, porque todo el mundo tenía que hacer algo. Por lo menos, me enseñaron a mezclar colores, las pegas, las fotos, eso estuvo medio raro porque no sé cómo todavía están ahí. La lechada negra, eso era enfangarse y el desastre que hubo al final se pudo arreglar. Porque hubo mucha lechada. Las personas que trabajamos fuimos bien amables. No tuve problemas con nadie. Me contó como horas, y eso me ayudó por otro lado.

Es importante resaltar como los jóvenes incluyen el asunto de la organización del grupo para el trabajo comunitario en sus relatos a la hora de expresar su sentimiento con el proyecto y su proceso. Eso denota la importancia de los proyectos colectivos en las vidas de los seres humanos. Otro de los jóvenes E.M profundiza sobre este particular:

Pues pienso que fue un esfuerzo. Trabajo en equipo. Un upgrade o mejoramiento en el área de los jóvenes, ya que nosotros no hacíamos casi nada. y esto fue una idea que nos llevó a sobre pasar las metas que nosotros queríamos. 
Añade Y.R:

Que está bien. Que la gente piensa que estamos haciendo algo por la comunidad. Nuevamente resalta la preocupación colectiva de aflora en como este grupo de jóvenes se planteó el proyecto de arte para su comunidad. La preocupación de romper con el mito de "los jóvenes están perdidos" fue motivación para la organización del grupo. Según S.P: Pues que es una buena idea. Es un ejemplo a seguir. De que no hay jóvenes perdidos y de que tienen talento. Cosas así.

Añade S.P:

Pues creo que se logró la admiración de la gente. El cambio de visión de "ahhh en Venezuela los jóvenes están perdidos. Pues porque aquí mucha gente decía ahh aquí no pasa nada. Pero al ver el mural dicen ahhh aquí hay gente que hace.

Proceso de organización del grupo. Las dinámicas del proceso de organización requieren un sinnúmero de asuntos que el individuo tiene que manejar en colectivo. La organización es necesaria para desarrollar grandes proyectos sociales. En el caso del proyecto de arte en la barriada Venezuela tuvo precisamente el objetivo de concretar una obra colectiva. E.M, nos relata:

Además de aprender y socializar con otras personas, a veces me sentía stresscico porque había que terminarlo en cuatro semanas, pero después me sentí más calmado. Digamos libre artísticamente. Pudimos utilizar cosas que nunca había utilizado. Tuve diversión. Logramos hacer la gente feliz. Trabaje con diferentes herramientas como la mezcla de lechada. Y me sentí lo mas bien.

En el caso de J.D, este nos comenta cómo fue el proceso de organización colectiva:

Este, bueno se logró todo, compartimos todos juntos, encontramos algo que todo el mundo está participando. En algún momento, nos tuvimos que ayudar todos. Aunque hubiera uno vago, todo el mundo quería hacer algo. Como que hubo una diferencia en las actitudes de todo el mundo.

Vemos en la descripción antes expuesta como los jóvenes tuvieron que salir de sus áreas de comodidad para cumplir el proyecto que pensaron en colectivo. Ante la pregunta; ¿el mural sirvió para unir el grupo?, el joven T.B señaló:

Sí. Yo diría que sí. Porque también uno de los propósitos era atraer a las personas y funcionó. Llegaron más personas.Añade N.O:

Bueno nosotros como grupo crecimos un montón. Nosotros nos unimos más. Antes era como... siempre fuimos unidos en una parte, pero el mural nos dio hasta más respeto mutuo. Con nuestro líder y con todos nosotros. Yo siento que nos unión un montón. Es que estuvimos un mes complejo ayudándonos y eso es como terapéutico.

Nuevamente, observamos como el sentimiento individual de satisfacción está atado a una lógica colectiva de un objetivo común. A su vez, como los jóvenes experimentaron un sentimiento de logro que los marcó profundamente. N.O nos relata:

Me marcó, porque soy la más grande de todos ellos. Y es como algo mas adentro. Uno crece de corazón, de nobleza, de estar con la gente, de ser amable con ellos, de aprender a bregar con ellos. De hablar con la gente. De ser carismática. Es algo más... es espiritual...

Estos sentimientos se manifiestan ya que no sólo la obra fue realizada por ellos/as, sino que el producto final fue pensado y diseñado por los/as jóvenes de la comunidad.

Planificación participativa. Escuchamos que cuando las personas piensan y profundizan hacen del proceso de análisis parte de su discurso de vida y por consecuente de sus acciones. Es por eso que la planificación participativa es muy importante en un proyecto como este. Veamos las respuestas de los/as jóvenes a la hora de pedirles que describieran el proceso de planificación de la obra de arte. N.O nos cuenta:

Pues la gente de CAUCE nos reunieron. Ya que teníamos un día que nos reuníamos en la semana y cada uno puso unos dibujos. Lo que ellos querían plasmar en el mural. Cualquier 
cosa. O sea que cada uno de los jóvenes puso su granito de arena. $Y$ ahí buscamos un artista que vio todo eso e hiciera algo. Y fue bien chévere porque no se quedó nada afuera. Estaba todo lo que todos querían y en sólo sketch. Nosotros estábamos como que waooo... mira la estrella. El sol, lo que nos representa a nosotros. Comenzamos a recoger las chapitas. Fuimos por todos los negocios de aquí. Le pedimos ayuda a uno de los dueños de negocios de aquí para que nos diera unas herramientas o materiales para seguir. También que un proceso de un mes completo. Todos los fines de semanas que estuvimos aquí. Dividiendo las cosa. Fue un bastante. Fue mucho. Marcó a todos nosotros. Porque sabíamos que era algo que era para nosotros y par dejarle a la comunidad. Era un orgullo venir a las 9am y trabajar, era chévere.

Añade S.P:

Bueno para mí, nosotros nos reunimos, planificamos las ideas y buscábamos ayudas. Así fuimos siendo más personas. O sea, entre nosotros, los jóvenes de la comunidad, teníamos que hacer algo que la gente se diera cuenta que los jóvenes de Venezuela estaban haciendo algo por el barrio. Para más decirte la mayoría de los que no son familia, no nos hablamos mucho. Sólo nos veíamos en la escuela. Ahora que estamos en el grupo hay mayor comunicación, mayor convivencia, como que más comunicación.

Lo importante para los jóvenes fue que en el producto final estuviera plasmado todas las ideas que juntos construyeron. La importancia la señala E.M cuando nos dice:

Pues primero todos nos sentamos juntos y hicimos el plan de lo que queríamos hacer. Dimos varias ideas y pues quisimos empezar primero con el mural porque queríamos buscar la forma de decirle a la gente que se quería hacer algo nuevo y se tomó la idea. Se logró hacer la idea. Tuvimos varias ayudas. Obviamente del artistas Lero Lero o Daniel. Y pues reunimos más jóvenes. Hicimos varias reuniones para que la comunidad se enterara y lazamos propaganda para poder realizar el proyecto. No sólo fue la comunidad y jóvenes del barrio, sino que también vinieron diferentes personas de distintas comunidades a ayudar.

Vemos que en el proceso no sólo los/as jóvenes trabajaron, sino que más gente su unió al esfuerzo. Es llevó a que los /as jóvenes dejaran de verse como actores pasivos en el proceso comunitario, a verse como agentes catalizadores para la acción comunitaria.

Liderato comunitario. Los proyectos se piensan, nacen y se construyen si existen manos que los concreten. Para eso es indispensable que hayan personas que sirvan de guías del proceso, por lo general se les llama líderes. Muchas personas piensan que jamás podrían asumir un proceso de liderato, sin embargo un proyecto como este les permitió a un grupo de jóvenes a desarrollar destrezas que les servirán para proyectos futuros en la vida. Además, fue la primera experiencia para realizar trabajo comunitario, cosa que ha demostrado ser importante en la vida en sociedad.

Ante la pregunta; ¿tú sientes que eres líder comunitario? Los siete entrevistados contestaron que sí. Además añadieron otros elementos que ponen de manifiesto el crecimiento del grupo como elemento importante en la barriada de Venezuela. Según N.O nos relata:

Al estar viendo, nos preguntaron qué otras cosa queríamos hacer después de esto y dimos nuestras ideas. $Y$ nos vieron como si fuéramos los futuros digamos líderes del barrio. Porque hicimos una diferencia. Ahora estamos con el plan de restaura el acueducto. Y ahí las personas nos consideran, como decir, como héroes de la historia.

De igual forma, T.B indica cómo el proyecto del mural sirvió de semilla para otros proyectos:

Pues nuestra próxima idea es bregar con el acueducto. No cambiarlo sino hacerlo una atracción. Para que las personas vean la importancia del acueducto. Porque la verdad eso es un National Tressure de América y es un lugar donde ocurrieron eventos históricos y queremos que las personas sepan que histórico tuvo eso. 
Añade S.P sobre la conclusión de los trabajos con el mural que:

Para mí las personas piensan bien. Que nosotros nos estamos esforzando, que nosotros queremos hacer un cambio. Que queremos cambiar la idea de la gente que vive aquí. Las personas vieron que trabajamos en equipo. Que somos unos jóvenes, que queremos un cambio para que la gente sepa que esto aquí no es malo. Vemos que no sólo las personas de la comunidad miran a los jóvenes como los futuros líderes, sino que también los jóvenes hicieron un esfuerzo por demostrar que los jóvenes no están perdidos y que pueden ser de gran influencia en la vida social de un país.

\section{Reflexiones finales}

Las verbalizaciones de los/as jóvenes, son un ejemplo de lo que podría suceder si nos enfocamos en abrir espacios para que la juventud se involucre en el quehacer social. Los/as jóvenes hablan, crean, planifican, coordinan y asumen posturas ante la realidad del país y sus espacios comunales. Su participación no se limita a "cuando las gallinas mean", y mucho menos cuando lo dicten los adultos, como tradicionalmente se concibe. Es por ello, que es de suma importancia provocar la inserción de los/ as jóvenes en los procesos organizativos y de acción dentro de las comunidades, y romper con toda relación de opresión y dominación que puede traer consigo el adultocentrismo.

En relación al arte, este trabajo demostró que es una excelente herramienta para fomentar y fortalecer la organización comunitaria, lo cual convierte a la manifestación del arte no en el objetivo principal, sino en un medio que da espacio a diversas dinámicas de solidaridad, empatía, organización y acción comunal. Y a su vez, expone a las personas de sectores populares a apreciar el arte y la cultura, no desde un sector privilegiado, sino desde sus propios espacios. Es por esto, que los/as profesionales del Trabajo Social deben ponderar la posibilidad de utilizar el arte en las comunidades, no limitándose a pequeños talleres temáticos, sino para el impacto de zonas maltrechas por el fenómeno de la migración, el abandono de espacios públicos y con el fin de organizar para que los/as ciudadanos/as demanden la intervención del Estado en espacios como éstos, los cuales crean problemas de salud y seguridad.

En el caso de la barriada Venezuela muchos/as de los/as jóvenes, percibieron la creación del mural, como una experiencia que los/as motivó a realizar otros proyectos en su comunidad. Es por eso, que el grupo se enfoca en su próximo plan, el cual busca rescatar los tanques del Acueducto Histórico de San Juan, localizados allí mismo. Esto representará no solo transformar el estado actual de una estructura abandonada, sino que resaltará la historia de la comunidad, provocará mayor organización comunitaria de los/as jóvenes y sobre todo, avivará el sentimiento de que un grupo de jóvenes pueda demostrar que la juventud no está perdida y que la misma con un poco de apoyo puede lograr asuntos trascendentales como lo es trabajar con un yacimiento arqueológico histórico.

El trabajo realizado en la barriada Venezuela ha llevado a que al momento de finalizar este escrito, CAUCE trabaje en la configuración de un grupo más amplio de líderes jóvenes de las comunidades de Buen Consejo, Venezuela, Santa Rita y Capetillo, quienes se proyectan como los/as futuros líderes de las comunidades riopedrenses. Estamos conscientes de las múltiples capacidades que poseen los/as jóvenes y por ende, nos enfocamos en seguir trabajando con el liderato juvenil.

\section{Referencias}

Alcatruz Riquelme, Paula. (2004). Aquí se pinta nuestra historia: el muralismo callejero como acercamiento metodológico al sujeto histórico poblador. Anuario de Pregrado, 1, (1-17).

Balardini, Sergio. (2005). ¿Qué hay de nuevo, viejo? Nueva Sociedad 200. pp. 96-107.

Burgos, Nilsa M. (2011). Investigación cualitativa: Miradas desde el Trabajo Social. Buenos Aires: Espacio Editorial.

De Pablos Ramírez, Carlos Juan, \& Sánchez
Tovar, Ligia. (2003). Estilos de vida y

revitalización del espacio urbano. Papers, 71, 13. Recuperado de:

http://www.raco.cat/index.php/papers/ article/viewFile/25753/25587

Fontánez Torres, Érika. (2014). Ambigüedad y Derecho: Ensayos de Crítica Jurídica. Cabo Rojo, Puerto Rico: Editorial Educación Emergente.

Krauskopf, Dina. (2005). Desafíos en la construcción e implementación de las 
políticas de juventud en América Latina. Nueva Sociedad 200. pp. 141 - 153.

Lalo, Eduardo. (2005). Los pies de San Juan. San Juan: Edición Tal Cual.

Lucca, Nydia, \& Berríos, Reinaldo. (2003). Descripción, análisis e interpretación: el modelo de Harry F. Wolcott. Investigación Cualitativa. Puerto Rico. Ediciones SM. pp. 485

Macassil Lavander, Sandro. (2001). Culturas Juveniles, Medios y Ciudadanía. El Nuevo Horizonte Generacional y las Disyuntivas de la Inserción de los Jóvenes en la Sociedad. Asociación de Comunicadores Sociales Calandria. Lima, Perú.

Martínez Vizcarrondo, Ana Maritza. (2009). La interdisciplinariedad en la formación del egresado y egresada de la Universidad de Puerto Rico: Una mirada desde la disciplina del trabajo social hacía otras disciplinas. Revista Análisis, 10(1), 167-187.

Nardone, Mariana. (2010).

Instituto de Investigación en Ciencias Sociales, 3(6). Recuperado de: h t t p : / / p3.usal.edu.ar/index.php/miriada/article/ view/24/59

Sánchez Tovar, Ligia, \& González, Carlos Juan. (2006). Una mirada teórica del Barrio. Estilos de vida y participación comunitaria. La calidad de vida como finalidad. pp. 20 - 28.

Palacios Garrido, Alfredo (2009). El arte comunitario: origen y evolución de las prácticas artísticas colaborativas. Arte terapia: papeles de arteterapia y educación artística

para la inclusión social, 4, 197-211. Recuperado de: $\quad$ ht t p : / / w w w . ub.edu/hsctreballsocial/sites/default/files/ pdfs/recursos/palacios_arte_comu nitario_origenes.pdf

Pérez y Pérez, Alfonso (s.f.). Escuela Mexicana de Pintura. Recuperado de: ht t p :// www.macay.org/exposicion/90/escuelamexicana-de-pintura

Pitts, Graham, \& Watt, David. (2001). The imaginary conference artwork magazine, 50, pp.7-14.

Pizarro, Doris. (2012). Construcción de Ciudadanía desde las luchas comunitarias en Puerto Rico: su dimensión política transformadora y la cuestión social. En R. Seda (Org.), Trabajo comunitario y descolonización. pp. 33-81. San Juan, Puerto Rico: Ediciones Callejón.

Quiles Rodríguez, Edwin. (2009). La ciudad de los balcones. Río Piedras, Puerto Rico: Editorial de la Universidad de Puerto Rico.

Rodríguez, Ernesto. (2005). Juventud, desarrollo y democracia en América Latina. Nueva Sociedad 200. pp. 52 - 69.

Roitter, Mario M. (2009). Prácticas Intelectuales Académicas y Extra-Académicas sobre Arte Transformador: Algunas Certezas y Ciertos Dilemas. pp.1-13.

Romo Rodríguez, María Paula. (2005). Testimonio: política desde I@s jóvenes, ¿nueva política? Nueva Sociedad 200. pp. 108 113.

Tonon, Graciela. (2009). Acerca de comprender el actual concepto de comunidad. En G. Tonon (Comp.), Comunidad, participación y socialización política. pp. 13-28. Buenos Aires, Argentina: Espacio Editorial.

Suess, Astrid. (2006). El arte como herramienta de transformación social: proyectos comunitarios. Encuentros conlaexpresión. pp.70-75.

Wolcott Harry F. (1994). Description, Analisys and Interpretation in Qualitative Inquiry. Transforming qualitive data. Description, analisys and interpretation. United States: Sage Publications. 